\title{
Electric Efficiency Modelling of a Complex Cogeneration Process Using Extreme Learning Machines
}

\author{
Sandra Seijo, Inés del Campo, Javier Echanobe, and Javier García-Sedano
}

\begin{abstract}
Extreme learning machine (ELM) has attracted increasing attention recently with its successful applications in classification and regression because it outperforms conventional artificial neural networks (ANN), and support vector machines (SVM) in some aspects. ELM provides a robust learning algorithm, free of local minima, without overfitting problems and less dependent on human intervention than the above methods. ELM is appropriate for the implementation of intelligent autonomous systems with real-time learning capability. Moreover, a number of complex industrial applications demanding a high performance solution could benefit from this approach.

This work proposes the modelling of a real complex cogeneration plant with the aim of obtaining higher energy production with a lower cost (i.e. maximum energy efficiency) using ELM. The accuracy and training time of the ELM-based model are compared with the results obtained using BP-ANN and SVM. ELM training is significantly faster than SLFNs and SVM while preserving the same accuracy level.
\end{abstract}

Index Terms-Modelling, extreme learning machine, cogeneration, efficiency.

\section{INTRODUCTION}

Artificial neural networks (ANN) have been widely used in a variety of engineering applications over the last two decades due to their ability: (1) to approximate complex nonlinear mappings directly from the input/output samples, and (2) to provide models for a large class of natural and artificial phenomena that are difficult to handle using classical parametric techniques. Many of these applications are based on single hidden-layer feedforward network topologies and gradient-descent training methods (GDM), mainly traditional backpropagation (BP) algorithm.

Although BP-based ANNs have been successfully applied to solve numerous problems, as much for classification [1] as for regression applications [2], they present some drawbacks that make them unsuitable for an increasing number of cutting-edge applications. It is well known that the design of BP based ANNs is a time-consuming task that depends on the skills of the designer to obtain effective solutions. The designer has to select the most suitable network parameters, optimize the parameters to avoid

Manuscript received February 9, 2015; revised June 15, 2015. This work was supported in part by the Basque Country Government under Grants IT733-13, and IG2012/221 (ICOGME), and the Zabalduz Program of the University of the Basque Country (Spain).

Sandra Seijo, Inés del Campo, and Javier Echanobe are with the Department of Electricity and Electronics, University of the Basque Country UPV/EHU Leioa, Vizcaya, Spain (e-mail: sandra.seijo@ehu.es, ines@we.lc.ehu.es, javi@we.lc.ehu.es).

Javier García-Sedano is with OPTIMITIVE S.L. Vitoria-Gasteiz, Álava, Spain (e-mail: javierg@optimitive.com). overfitting, and be aware of local minima. As a consequence, applications requiring autonomy (i.e. no human intervention) are difficult to manage using this approach. Another mature machine learning technique is support vector machine (SVM) introduced by Vapnik [3]. SVM is free of local minimum, and is able to improve the generalization performance of traditional ANNs for some important application domains (e.g. machine vision, handwritten character recognition, medicine and bioinformatics applications, among others). However, they present some drawbacks as a time-consuming training algorithm difficult to tune without human intervention.

Extreme learning machine (ELM) is a novel learning algorithm for training single hidden-layer feedforward neural networks (SLFNs) [4], [5]. It randomly chooses the input weights of the hidden-layer neurons and analytically determines the output weights through simple matrix computations, therefore featuring a much faster learning algorithm than most popular learning methods such as backpropagation [6]. ELM is based on a simple tuning-free algorithm and parameter selection is not required. Besides, learning with ELM does not present local minima or overfitting problems. All these characteristics makes ELM very useful dealing with real-life applications where autonomous control systems and fast adaptation are necessary [7]. A proper example of this kind of applications are complex industrial processes where the efficiency of the process is to be modelled and optimized in real-time.

In this work, the modelling of the effective electric efficiency $\left(\xi_{\mathrm{EE}}\right)$ of a real complex combined heat and power (CHP) process using ELM is proposed. The CHP plant generates electricity with four internal combustion engines and a steam turbine. The energy is sold and the heat from the engines is used to generate more energy in the steam turbine and in a slurry drying process. To achieve this modellization, the plant is separated into different parts, and a model is generated for each subprocess using data sets from the real plant. Finally, the ELM models (i.e. accuracy, speed and topology) are compared with BP-ANN and SVM models to demonstrate the effectiveness of the ELM approach.

\section{EXTREME LEARNING MACHINE}

Extreme learning machine was originally proposed by Huang et al. [6] for the single hidden-layer feedforward neural networks and then extended to the generalized single hidden-layer feedforward networks where the hidden layer needs not be neuron alike [8].

Suppose a SLFN with $\mathrm{n}$ inputs, $\mathrm{m}$ outputs and 1 nodes in the hidden layer (see Fig. 1). The output $j$ of the SLFNs can 
be written as:

$$
y_{j}=\beta_{j} G
$$

where $\beta_{j}=\left[\beta_{j 1}, \ldots, \beta_{j l}\right]$ is the weight vector connecting the hidden layer and the $\mathrm{j}$ th output node, $G=\left[G_{1}, G_{i}, \ldots, G_{l}\right]$ is the vector formed by the values $G_{i}=g\left(a_{i} x+b_{i}\right)$ being $\mathrm{g}()$ the activation function, $a_{i}=\left[a_{i 1}, \ldots, a_{i n}\right]^{T}$ the vector connecting the input $x=\left[x_{1}, \ldots, x_{n}\right]$ with the $i$ th hidden node and $b_{i}$ the bias of the $i$ th hidden node.

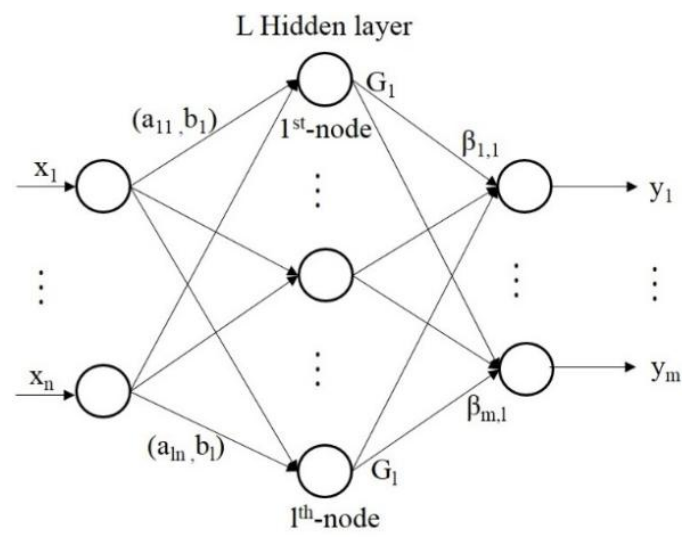

Fig. 1. The topological structure of the SLFN.

The main difference between ELM and traditional learning approaches is that the hidden layer need not be tuned; it is a randomized layer. That is to say, the set of parameters of the hidden nodes $\left(a_{i}, b_{i}\right), 1 \leq i \leq 1$, are randomly generated. Therefore, they are independent of the application and of the training samples. Learning in ELM is a straightforward procedure that aims at computing the vector of output weights, $\beta_{j}$ in (1), for each output node.

For $k=1, \ldots, K$ arbitrary distinct samples $\left(x^{k}, t^{k}\right)$, where $x^{k}=\left[x_{1}^{k}, \ldots, x_{n}^{k}\right]^{T} \in R^{n}$ are the input data and $t^{k}=$ $\left[t_{1}^{k}, t_{2}^{k}, \ldots, t_{m}^{k}\right]^{T} \in R^{n}$ are the target data, the above linear equations can be written in the matrix form:

$$
H \beta=T
$$

where $H\left(a_{1}, \ldots, a_{n}, b_{1}, \ldots, b_{l}, x^{1}, \ldots, x^{K}\right)$

$$
H=\left[\begin{array}{ccc}
G\left(a_{1} \cdot x^{1}+b_{1}\right) & \cdots & G\left(a_{l} \cdot x^{1}+b_{l}\right) \\
\vdots & \ddots & \vdots \\
G\left(a_{1} \cdot x^{K}+b_{1}\right) & \cdots & G\left(a_{l} \cdot x^{K}+b_{l}\right)
\end{array}\right]
$$

$T=\left[t_{1}, \ldots, t_{k}\right]^{\prime}$ is a vector of target labels and $\beta=$ $\left[\beta_{1}, \ldots, \beta_{m}\right]^{\prime}$. The solution of above equation is given as: $\beta=H^{\dagger} T$, where $H^{\dagger}$ is the Moore-Penrose generalized inverse of matrix $\mathrm{H}$ [9].

\section{COGENERATION Plant}

The CHP plant being evaluated is located in Monzón (Huesca), in the North of Spain. The plant generates electricity with four internal combustion engines (nominal power of each being $3700 \mathrm{~kW}$ ) feeded with natural gas and a steam turbine. The engines are refrigerated with two refrigeration circuits that use water from the cooling towers as Fig. 2 shows. Therefore, the engines generate electrical energy and high temperature gases. Subsequently, the electrical power generated is sold and the high temperature gases go to an exhaust steam boiler. Moreover, the steam generator creates steam using the heat from the exhaust steam boiler. This steam is used in a steam turbine to generate more electricity, with $1000 \mathrm{~kW}$ of nominal power that is also sold. The heat from exhaust steam boiler is also used in a slurry drying process that uses the slurry from nearby farms. After being processed by the plant it becomes fertilizer and clean water for irrigation.

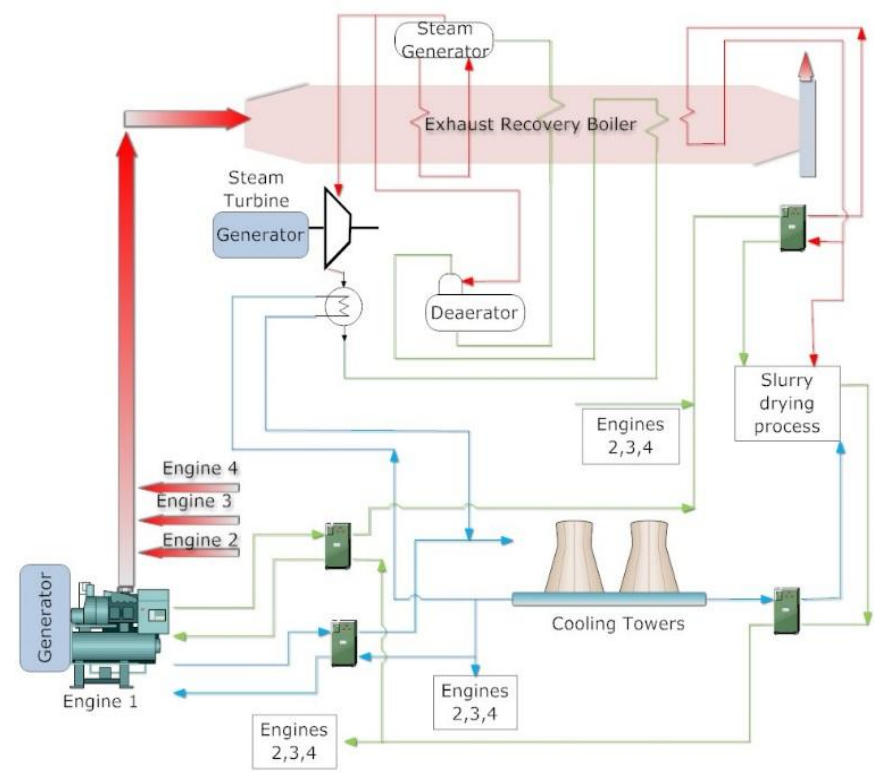

Fig. 2. Scheme of the cogeneration process.

The effective electric efficiency of the plant is defined as:

$$
\xi_{E E}=\frac{W_{E}}{Q_{F U E L}-\sum\left(\frac{Q_{T H}}{\alpha}\right)} \cdot 100
$$

where $W_{E}$ is the net useful power output generated by the four engines and the steam turbine, $Q_{F U E L}$ is the total fuel (natural gas) input used by the four engines, $Q_{T H}$ is the sum of the net useful thermal outputs (related with the useful thermal energy used in the slurry process) and $\propto$ is a bonus factor, in this CHP process equal to 0.9.

Table I shows the different parts of the CHP plant together with the corresponding models and the involved input/output variables.

TABLE I: MODELS DEVELOPED AND RELATED VARIABLES

\begin{tabular}{lccc}
\hline \hline Cogeneration Part & Models & Inputs & Output \\
\hline Cooling circuits & Cooling & 3 inputs & Temperature \\
& $\begin{array}{c}\text { A/B/C/D } \\
\text { Engine }\end{array}$ & 2 inputs & Fuel flow \\
Engines & $\mathrm{A} / \mathrm{B} / \mathrm{C} / \mathrm{D}$ & 2 inputs & Steam flow \\
Recovery Boiler & & 2 inputs & Pressure \\
Steam Turbine Condenser & 3 inputs & Power \\
Steam Turbine & 5 inputs & Flow \\
Slurry drying process & &
\end{tabular}

\section{EXPERIMENTATION AND RESULTS}

In this section, the development of a model of the real cogeneration plant using ELM is presented. To do this, the 
plant is separated into different parts (see Table II). A dataset with 211 variables collected over a one-year period from the whole cogeneration process is available. Data mining techniques are applied to choose the meaningful variables involved in the modelling (inputs and targets) and to obtain a proper dataset without outliers, missing data or un-informative variables. Finally, a training set and a testing set with almost 19000 data each one are obtained.

TABLE II: RESULTS AND CHARACTERISTICS OF THE MODELS

\begin{tabular}{|c|c|c|c|c|}
\hline & Parameter & ELM & ANN-BP & SVM \\
\hline \multirow{5}{*}{$\begin{array}{l}\text { Irrigation } \\
\text { Engine A }\end{array}$} & Training MSE & 0.0314 & 0.0465 & 0.0243 \\
\hline & Testing MSE & 0.0504 & 0.0507 & 0.0539 \\
\hline & Training Time (s) & 0.0624 & 14.7109 & 10.4833 \\
\hline & Testing Time (s) & $<10^{-4}$ & 0.0312 & 4.2432 \\
\hline & No. of nodes & 18 & 20 & 7669 \\
\hline \multirow{5}{*}{$\begin{array}{l}\text { Irrigation } \\
\text { Engine B }\end{array}$} & Training MSE & 0.1530 & 0.2286 & 0.1683 \\
\hline & Testing MSE & 0.1649 & 0.2107 & 0.2139 \\
\hline & Training Time (s) & 0.2184 & 8.2837 & 11.3257 \\
\hline & Testing Time (s) & $<10^{-4}$ & 0.0312 & 5.6472 \\
\hline & No. of nodes & 35 & 10 & 12236 \\
\hline \multirow{5}{*}{$\begin{array}{l}\text { Irrigation } \\
\text { Engine C }\end{array}$} & Training MSE & 0.2887 & 0.0992 & 0.0673 \\
\hline & Testing MSE & 0.2845 & 0.1313 & 0.1970 \\
\hline & Training Time (s) & 0.1716 & 9.5317 & 12.7141 \\
\hline & Testing Time (s) & $<10^{-4}$ & 0.0312 & 5.1168 \\
\hline & No. of nodes & 10 & 28 & 10434 \\
\hline \multirow{5}{*}{$\begin{array}{l}\text { Irrigation } \\
\text { Engine D }\end{array}$} & Training MSE & 0.4104 & 0.4324 & 0.4251 \\
\hline & Testing MSE & 0.2911 & 0.2973 & 0.4896 \\
\hline & Training Time (s) & $<10^{-4}$ & 5.6472 & 9.9061 \\
\hline & Testing Time (s) & $<10^{-4}$ & 0.0156 & 1.794 \\
\hline & No. of nodes & 13 & 5 & 13607 \\
\hline \multirow{5}{*}{$\begin{array}{c}\text { Recovery } \\
\text { Boiler }\end{array}$} & Training MSE & 0.3407 & 0.3687 & 0.2787 \\
\hline & Testing MSE & 0.5157 & 0.6036 & 0.5449 \\
\hline & Training Time (s) & $<10^{-4}$ & 5.0856 & 16.0057 \\
\hline & Testing Time (s) & $<10^{-4}$ & 0.0468 & 5.7876 \\
\hline & No. of nodes & 4 & 5 & 13607 \\
\hline \multirow{5}{*}{$\begin{array}{c}\text { Steam } \\
\text { Turbine } \\
\text { Condenser }\end{array}$} & Training MSE & 0.3728 & 0.2478 & 0.1634 \\
\hline & Testing MSE & 0.2979 & 0.2751 & 0.3159 \\
\hline & Training Time (s) & $<10^{-4}$ & 16.1617 & 4.6332 \\
\hline & Testing Time (s) & $<10^{-4}$ & 0.0312 & 1.794 \\
\hline & No. of nodes & 4 & 5 & 2982 \\
\hline \multirow{5}{*}{ Engine A } & Training MSE & 0.2927 & 0.1897 & 0.2047 \\
\hline & Testing MSE & 0.2928 & 0.2933 & 0.2879 \\
\hline & Training Time (s) & 0.1872 & 31.5434 & 18.9073 \\
\hline & Testing Time (s) & 0.0624 & 0.0312 & 8.2837 \\
\hline & No. of nodes & 38 & 10 & 12860 \\
\hline \multirow{5}{*}{ Engine B } & Training MSE & 0.2133 & 0.2116 & 0.1699 \\
\hline & Testing MSE & 0.1934 & 0.2181 & 0.2098 \\
\hline & Training Time (s) & 0.2340 & 9.9373 & 11.1477 \\
\hline & Testing Time (s) & 0.0624 & 0.0312 & 7.9405 \\
\hline & No. of nodes & 39 & 5 & 12456 \\
\hline \multirow{5}{*}{ Engine $\mathrm{C}$} & Training MSE & 0.2887 & 0.2324 & 0.2253 \\
\hline & Testing MSE & 0.2845 & 0.2929 & 0.3609 \\
\hline & Training Time (s) & 0.1716 & 12.1681 & 14.1493 \\
\hline & Testing Time (s) & 0.0624 & 0.0468 & 8.9545 \\
\hline & No. of nodes & 28 & 15 & 13357 \\
\hline Engine D & Training MSE & 0.1595 & 0.2045 & 0.1478 \\
\hline
\end{tabular}

\begin{tabular}{ccccc}
\hline & Testing MSE & 0.2210 & 0.2480 & 0.3380 \\
& Training Time (s) & 0.546 & 15.0541 & 12.6921 \\
& Testing Time (s) & 0.1404 & 0.0312 & 7.8781 \\
& No. of nodes & 85 & 20 & 12089 \\
\hline \multirow{4}{*}{ Steam } & Training MSE & 0.1299 & 0.2448 & 0.2484 \\
Turbine & Testing MSE & 0.2659 & 0.3314 & 0.3024 \\
& Training Time (s) & 0.0624 & 9.6097 & 15.2881 \\
& Testing Time (s) & $<10^{-4}$ & 0.0156 & 6.7704 \\
& No. of nodes & 8 & 10 & 14433 \\
& & & & \\
\hline \multirow{4}{*}{ Slurry } & Training MSE & 0.1123 & 0.1434 & 0.0604 \\
drying & Testing MSE & 0.0759 & 0.3645 & 0.1306 \\
process & Training Time (s) & 0.3352 & 5.0856 & 13.7749 \\
& Testing Time (s) & $<10^{-4}$ & 0.0468 & 6.1308 \\
& No. of nodes & 11 & 5 & 12105 \\
\hline \hline
\end{tabular}

To model the cogeneration plant, each model is trained with the ELM algorithm (the number of hidden nodes from 1 up to 100). Finally, the best performance of 10 trials of simulations for each model is selected. The experiments were carried out using Matlab tool.

For comparison purpose, we have also implemented both the ANN-BP with a single hidden-layer, and the SVM using radial basis function kernel. The same topology as in the case of ELM has been used for BP-ANN modelling, but the number of hidden nodes are gradually increased by an interval of 5 up to 100 , and 3000 epoch are selected in each training. For the SVM the cost parameter $C$ has been chosen equal to the range of output values of training data [10]. The kernel parameter $\gamma$ and the intensive zone $\varepsilon$ values are selected from the best accuracy for the combination of: $\gamma=\left[2^{-7}, 2^{-6}, \ldots, 2^{7}\right]$, and $\varepsilon=[0.1,0.2, \ldots, 0.5]$. SVM models are carried out using LIBSVM [11]. Table II compares the overall results among all the models. The table presents the training and testing accuracy (normalized mean square error (MSE)), training and testing time, and number of nodes created.

General speaking, all algorithms provide approximately the same accuracy and obtain good performance. However, the difference between training and testing accuracy has a larger difference in most of cases for ANN and SVM than in ELM model. This can be explained by the fact that ANN and SVM tends to overfitting the training data. The generalization performance of ELM is very stable on a wide range of number of hidden nodes, as Fig. 3 shows for the four engine models.

On the other hand, ELM needs more hidden nodes than ANN to reach a similar performance, while SVM requires much more nodes than ELM and ANN. Regarding the training time, ELM is the fastest learning algorithm in all the cases, with training time hundreds of times faster than ANN and SVM. Also ELM is the fastest algorithm for testing (response time to unknown data set for testing).

The effective electric efficiency of the plant is calculated using Equation (4). For this purpose, firstly the real effective electric efficiency in each sample of the dataset is calculated using real values. To obtain $W_{E}$ the real values of the power generated by the four engines and the steam power are used To obtain $Q_{F U E L}$ the real natural gas flow used by each engine is used, and for $Q_{T H}$ the real thermal energy used in 
the slurry process is calculated taken into account the amount of slurry dried in each sample of the dataset. Afterward, the effective electric efficiency is obtained in the same way, but with the predicted values obtained with the ELM models, i.e. the power of the steam turbine used is the predicted value for the steam turbine model, the natural gas flow is the predicted value for each engine ELM model, and the thermal energy in the slurry drying process is obtained from the prediction of the slurry drying process ELM model. To illustrate the result of ELM modelling, Fig. 4 shows the effective electric efficiency using Equation (4) with real data and using the predictions of the ELM models. As can be seen, expected efficiency is able to follow the trend of the real efficiency.

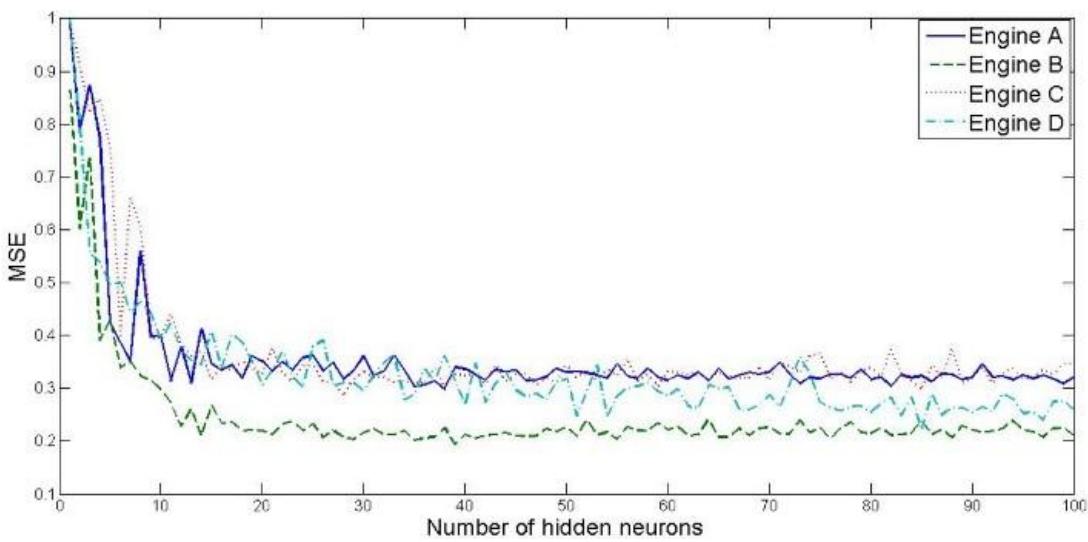

(a) Performance of ELM according to the number of hidden neurons

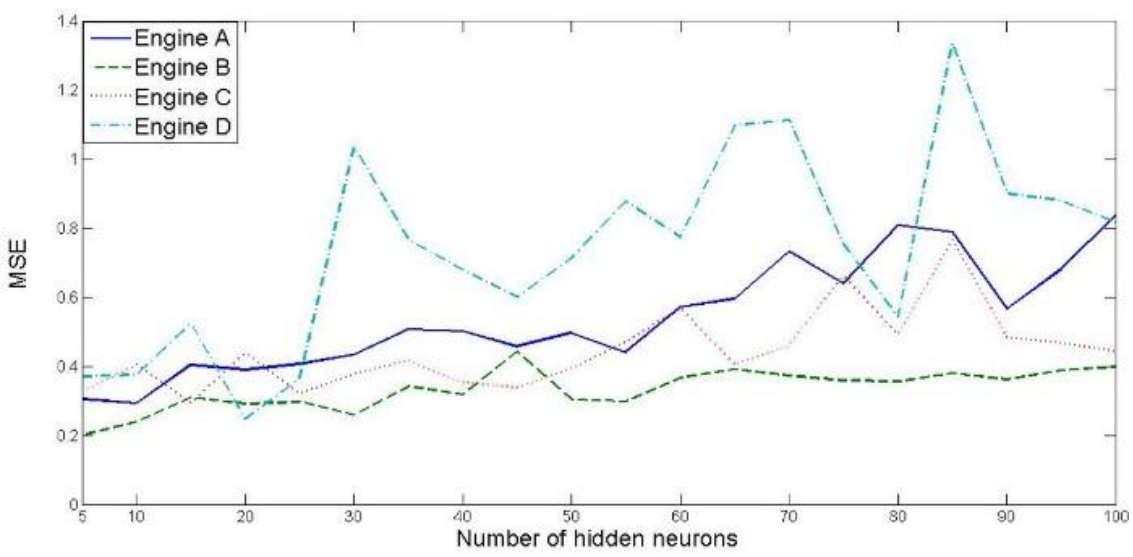

(b) Performance of ANN-BP according to the number of hidden neurons

Fig. 3. Performance of ELM and BP according to the number of hidden neurons.

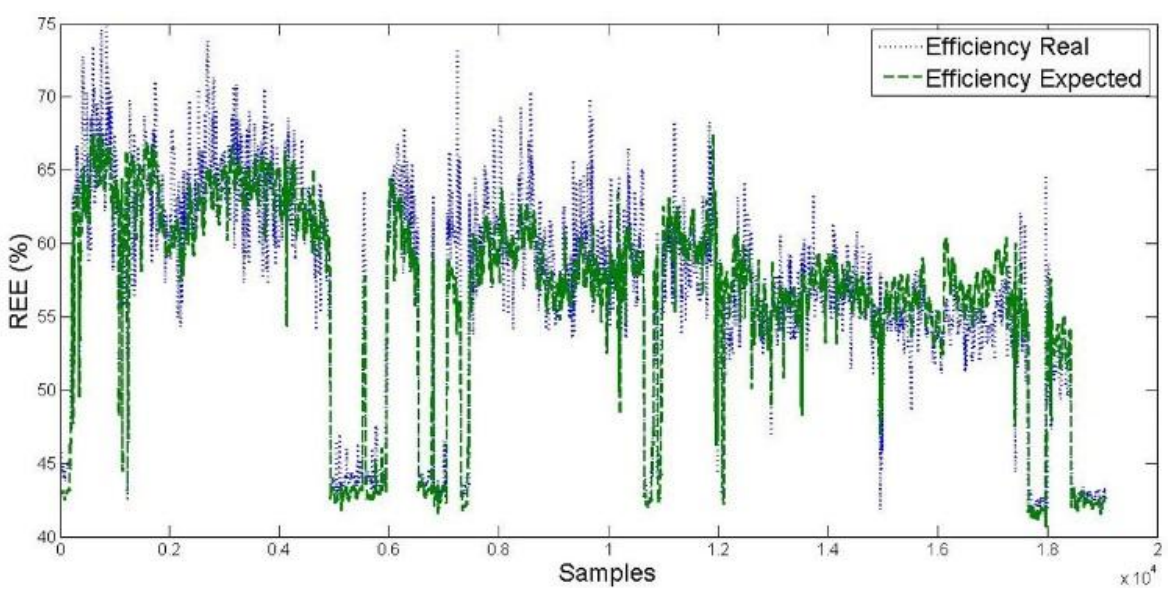

Fig. 4. Results of the ELM models for the effective electric efficiency.

\section{CONCLUSION}

In this work, the modelling of the effective electric efficiency of a real complex cogeneration process using ELM is proposed. To achieve this, the plant is separated into different parts and a model is generated for each subprocess using data sets from the real plant. With the same data sets and setting variables, models are also constructed using
ANN trained with BP-GDM and SVM using radial basis function kernel.

Although all algorithms show proper testing accuracy, the large difference between training and testing accuracy in ANN and SVM reveals overfitting problems. The number of nodes in ELM and ANN models are similar (slightly lower for ANN models). Moreover ELM is very stable in a wide range of number of hidden nodes. Experimental results also 
show that ELM is largely the fastest of all, spending hundreds of times less than SVM and ANN.

As a conclusion of the experimental results, ELM provides a robust learning algorithm, free of local minima, without overfitting problems. ELM algorithm is very fast learning and less dependent on human intervention than the ANN-BP or SVM. All this characteristics make ELM suitable for autonomous real-time monitoring of the plant.

\section{REFERENCES}

[1] Y. Li, B. Yang, Z. Wang, and X. Wang, "Fault pattern classification of turbine-generator set based on artificial neural network," in Proc. the International Conference on Computer Application and System Modeling, 2010, vol. 15.

[2] H. Nikpey, M. Assadi, and P. Breuhaus, "Development of an artificialneural network model for combined heat and power micro gas turbines," in Proc. International Symposium on Innovations in Intelligent Systems and Applications, 2012, pp. 1-5.

[3] C. Cortes and V. Vapnik, "Support-vector networks," Machine Learning, vol. 20, no. 3, pp. 273-297, September 1995.

[4] G.-B. Huang, D. Wang, and Y. Lan, "Extreme learning machines: A survey," International Journal of Machine Learning and Cybernetics, vol. 2, no. 2, pp. 107-122, 2011.

[5] G.-B. Huang, H. Zhou, X. Ding, and R. Zhang, "Extreme learning machine for regression and multiclass classification," IEEE Transactions on Systems, Man, and Cybernetics, Part B: Cybernetics, vol. 42, no. 2, pp. 513-529, 2012.

[6] G.-B. Huang, Q.-Y. Zhu, and C.-K. Siew, "Extreme learning machine: Theory and applications," Neurocomputing, vol. 70, no. 1-3, pp. 489-501, 2006.

[7] J. E. R. Finker, I. del Campo, and V. Martínez, "An intelligent embedded system for real-time adaptive extreme learning machine," in Proc. 2014 IEEE Symposium on Intelligent Embedded Systems 2014, pp. 61-69.

[8] G.-B. Huang and L. Chen, "Convex incremental extreme learning machine," Neurocomputing, vol. 70, no. 16-17, pp. 3056-3062, 2007.

[9] D. Serre, Matrices: Theory and Applications, Springer, 2001, ch. 8, pp. $145-147$

[10] D. Mattera and S. Haykin, "Advances in kernel methods," in Support Vector Machines for Dynamic Reconstruction of a Chaotic System, B. Schölkopf, C. J. C. Burges, and A. J. Smola, Eds. Cambridge, MA, USA: MIT Press, 1999, pp. 211-241.

[11] C.-C. Chang and C.-J. Lin, "LIBSVM: A library for support vector machines," ACM Transactions on Intelligent Systems and Technology, vol. 2, no. 3, 2011.

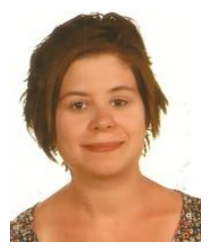

Sandra Seijo was born in Leon, Spain, in 1987. She received the Dipl. Ing. degree in industrial chemistry, and the M.Sc. degree from the University of Valladolid (UVA), in 2010 and 2012, respectively. She is a predoctoral researcher (granted by the Basque Government, Spain) from 2012. She is with the Department of Electricity and Electronics in the University of the Basque Country (UPV/EHU), Bilbao, Spain. Her research interests focus on 1) computational intelligence: artificial neural networks, fuzzy systems, and neuro-fuzzy systems; 2) Data
Mining and modelling of complex industrial processes; 3) Optimization of complex industrial processes for maximum energy efficiency.

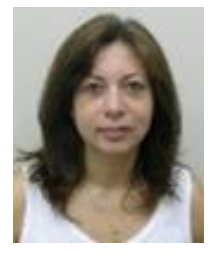

Inés del Campo was born in Buenos Aires, Argentina, in 1961. She received the Licenciado degree in physics with specialization in electronics and automatics in 1987 and the Ph.D. degree in physics in 1993, both from the University of the Basque Country (UPV/EHU), Bilbao, Spain. Currently she is a senior lecturer in the Electricity and Electronics Department of the Faculty of Sciences and Technology of the UPV/EHU. She has published articles in international journals and conferences in the areas of electronics, computational intelligence, intelligent control, ambient intelligence, and pattern recognition, among others. Her research interests mainly concern system-on-chip (SOC) design, hardware/software codesign, reconfigurable hardware, pervasive computing, artificial neural networks (ANNs), fuzzy systems, and genetic algorithms. She is also interested in the internet of things and its application in the context of ubiquitous computing and ambient intelligence.

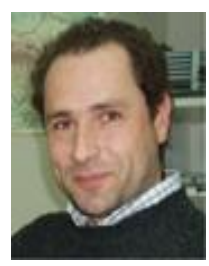

Javier Echanobe received the licenciado degree in physics from the University of the Basque Country (UPV/EHU), Spain, and the Ph.D. degree from the University of Navarra, Pamplona, Spain, in 1990 and 1998, respectively. He was a predoctoral researcher (granted by the Basque Government) from 1992 to 1996. He has been an associate professor in the Department of Electricity and Electronics, UPV/EHU, from 1999 to 2009. Since 2009 he is a Senior Lecturer in that department His research interests focus on 1) digital electronics: embedded systems, reconfigurable FPGAs, DSPs, SoPC; 2) computational intelligence: artificial neural networks, fuzzy systems, and neuro-fuzzy systems; 3) ubiquitous computing: ambient intelligence, intelligent environments. Dr. Echanobe has published many papers in international journals and conferences in most of those areas.

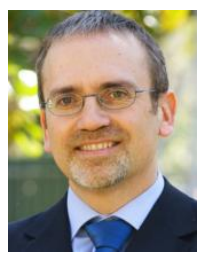

Javier García-Sedano received the licenciado degree in physics, specialised in electronics and automation, from the University of the Basque Country (UPV/EHU), Spain. The areas of his work and expertise are in knowledge-based applications in manufacturing; hybrid symbolic-genetic learning techniques for industrial process optimisation; intelligent agents for industrial applications; semantic-based product management and configuration. With more than 26 years of experience in these fields, he is the CEO and Founder of the OPTIMITIVE Group, with a large track of successful innovative Artificial Intelligence applications in Industry. He worked previously in companies like IBERDROLA Engineering, ORMAZABAL Group and TECNALIA Research Corporation, having carried out RTD and Innovation Management responsibilities, achieving successful technology transfer to the industry in the mentioned fields of specialisation. 